\title{
MALLET THUMB
}

\author{
K. M. DIN, B. F. MEGGITT
}

\section{From the Luton and Dunstable Hospital, Luton, and Addenbrooke's Hospital, Cambridge}

\begin{abstract}
The uncommon injury of mallet thumb is described, with four case reports. In diagnosis the condition must be distinguished from dropped thumb. Operative treatment is advised which, in this small series, gave excellent results.
\end{abstract}

Compared with mallet finger, mallet thumb is distinctly uncommon. The following four cases therefore seem worth reporting. They were collected over a period of four years; in the same period 48 cases of mallet finger were treated.

Case 1. A 33-year-old man presented at the accident department with a mallet thumb; he was right-handed and worked as a computer programmer. He had been kicked on the tip of his left thumb during a game of rugby four days previously.

On examination, there was swelling and tenderness of the distal part of the dorsum of the left thumb. There was a flexion deformity of $\mathbf{4 0}$ degrees at the interphalangeal joint (Fig. 1) but a full range of passive extension. Radiographs showed no evidence of bony injury (Fig. 2).

At operation, the extensor pollicis longus tendon was found to have been completely avulsed from its insertion; there was a gap of one centimetre between the end of the tendon and the site of insertion. The tendon was reattached to the base of the distal phalanx by passing $2 / 0$ silk mattress sutures through holes drilled in the bone. The thumb was splinted in extension for three weeks in a forearm plaster-cast, then mobilised.

Eight weeks after operation he had a range of active flexion and extension of 0 to 45 degrees at the interphalangeal joint, and was able to resume his normal duties.

Case 2. A 39-year-old man presented at the accident department; he was right-handed and worked in a factory. He had trapped his right thumb between two wheelbarrows at work four hours previously. On examination, the right thumb was swollen, tender and deformed. Radiographs showed an oblique fracture of the middle of the shaft of the proximal phalanx, with displacement. An attempted closed manipulation was unsuccessful. At open reduction the extensor pollicis longus tendon was found to have ruptured just proximal

K. M. Din, FRCS, Senior Orthopaedic Registrar

Luton and Dunstable Hospital, Lewsey Road, Luton, Bedfordshire, England.

B. F. Meggitt, FRCS, Consultant Orthopaedic Surgeon

Addenbrooke's Hospital, Hills Road, Cambridge CB2 2QQ, England. Requests for reprints should be sent to Mr K. M. Din.

(C) 1983 British Editorial Society of Bone and Joint Surgery $0301-620 \mathrm{X} / 83 / 5127 \$ 2.00$ to its insertion. The proximal part was caught on the spikes at the site of fracture, preventing reduction. Once the tendon was freed, the fracture was easily reduced, but was unstable. It was fixed internally using two parallel Kirschner wires. Then the extensor tendon was repaired with $2 / 0$ silk mattress sutures. The thumb was splinted in extension in a forearm plaster-cast for four weeks. The two Kirschner wires were then removed and active mobilisation of the thumb started. Eight weeks after operation there was radiographic consolidation of the fracture in a satisfactory position. The range of active flexion and extension at the interphalangeal joint was 0 to 25 degrees and the patient was able to resume his normal work.

Case 3. A 29-year-old farmer was leading a pig with a rope wrapped around his right hand. The pig suddenly bolted and jerked the rope, resulting in painful forced flexion of the farmer's thumb. He presented with a mallet thumb deformity. The right thumb was tender and swollen over the dorsum; active extension at the interphalangeal joint was absent. There was weak but full extension at the metacarpophalangeal joint. Radiographs showed no evidence of bony injury.

At operation, the extensor pollicis longus tendon was found to be ruptured one centimetre proximal to its insertion with a gap of 0.75 centimetres between the fairly clean ends. It was possible to bring the two ends together only by exerting traction on the proximal end and with the thumb in extension. The tendon was repaired with $2 / 0$ silk mattress sutures. The thumb was splinted in extension in a forearm plaster-cast for three weeks and then mobilised. Eight weeks after operation good function had been restored with strong active extension at the interphalangeal joint and a range of active flexion and extension of 0 to 45 degrees.

Case 4. A 54-year-old sales engineer presented with a mallet thumb. He had cut the dorsum of his left thumb while carving a joint of meat an hour previously.

On examination, there was a small incised wound over the dorsum of the thumb distal to the metacarpophalangeal joint. There was no active extension at the interphalangeal joint and a mallet deformity was present. The metacarpophalangeal joint showed a weak but full 
range of active extension. At operation the extensor pollicis longus tendon was found to be completely divided one centimetre distal to the metacarpophalangeal joint with a gap of 0.5 centimetres between the two cleanly divided ends. The tendon was repaired with $2 / 0$ silk mattress sutures. The thumb was splinted in extension in a forearm plaster-cast for four weeks and then mobilised. Eight weeks after operation there was strong active extension at the interphalangeal joint and a range of active flexion and extension of 0 to 45 degrees. The patient resumed his work as a sales engineer.

\section{DISCUSSION}

The terms "dropped" and "mallet" are unsatisfactory because there is confusion regarding their use. It is suggested that the term "mallet" should be used for the flexion deformity at the interphalangeal joint of the thumb (or the distal interphalangeal joint of a finger) when this deformity follows loss of continuity of the extensor mechanism at or close to its insertion. The term "dropped" should be reserved for loss of continuity of the extensor mechanism proximal to the metacarpophalangeal joint of the thumb or finger; the term "dropped" is here preferred because it describes the position of the whole finger or thumb at the metacarpophalangeal joint.

The differential diagnosis between mallet (Figs 1 and 2) and dropped thumb can be made on the basis of the history of the injury, the clinical examination and the radiographic findings. In a neglected case, and where the injury was a closed one, differentiation may not be easy.

A history of injury distal to the metacarpophalangeal joint, tenderness at or close to the insertion of the extensor pollicis longus tendon and radiographic evidence of injury to the base of the distal phalanx, favour the diagnosis of mallet thumb.

The diagnosis of dropped thumb is suggested by the presence of a flexion deformity at both the metacarpophalangeal and the interphalangeal joints; the scar of any injury is proximal to the metacarpophalangeal joint. Active extension is usually absent at both the metacarpophalangeal and interphalangeal joints, but if the extensor pollicis brevis is intact, weak extension at the metacarpophalangeal joint may be present. A dropped thumb that has been left untreated may, after a few days,

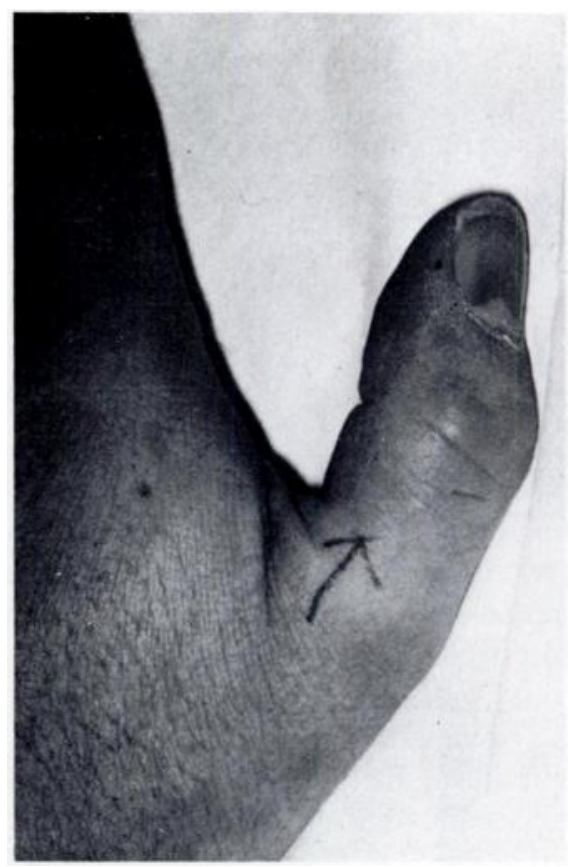

Fig. 1

Clinical photograph and radiograph of the left thumb showing a mallet deformity.

develop "trick" extension; this "trick" movement at the interphalangeal joint is due to the intact expansions which run from the abductor pollicis brevis and adductor pollicis to the extensor hood of the extensor pollicis longus; these expansions then continue and are inserted into the dorsal aspect of the base of the distal phalanx of the thumb.

Current literature gives little guidance to the treatment of mallet thumb. We advocate operative treatment of mallet thumb for the following reasons. (1) There are no reports of successful conservative treatment of mallet thumb and the results of the splinting of mallet finger are not always satisfactory. (2) Unlike the thin frayed extensor tendon in mallet finger the tendon in mallet thumb is thicker and holds sutures well. (3) The presence of a fairly large gap due to retraction of the proximal end of the extensor pollicis longus tendon in this series suggests that conservative treatment alone would not give a good result in this essential digit. With the excellent results of this small series, we therefore advise primary repair of mallet thumb.

The authors wish to thank Mr Beard, of Addenbrooke's Hospital, Cambridge, and Mr Harrison of the Luton and Dunstable Hospital, for the photographs. 